RAIRO Operations Research

RAIRO Oper. Res. 35 (2001) 143-163

\title{
MULTICRITERIA SCHEDULING PROBLEMS: A SURVEY
}

\author{
V. T'KINDT ${ }^{1}$ AND J.-C. BILlaUt ${ }^{1}$
}

\begin{abstract}
This paper presents a state-of-the-art survey on multicriteria scheduling and introduces a definition of a multicriteria scheduling problem. It provides a framework that allows to tackle multicriteria scheduling problems, according to Decision Aid concepts. This problem is decomposed into three different problems. The first problem is about obtaining a model. The second one is how to take criteria into account and the third one is about solving a scheduling problem. An extension to an existing notation for scheduling problems is proposed for multicriteria scheduling problems. Then, basic results from the literature on multicriteria optimization are presented. These results are used to build the final scheduling problem to solve. Finally a survey is presented for one-machine, parallel machines and flowshop multicriteria scheduling problems.
\end{abstract}

Résumé. Ce travail présente un état de l'art sur les problèmes d'ordonnancement multicritères et introduit une nouvelle définition de ces problèmes. Nous proposons également une démarche, conforme aux principes de l'aide multicritère à la décision, pour aborder les problèmes d'ordonnancement multicritères. Ces problèmes sont décomposés en trois sous-problèmes. Le premier concerne la modélisation du problème d'ordonnancement considéré. La résolution du second sousproblème conduit à répondre à des questions : comment prendre en compte les critères pour calculer des optima de Pareto ? Quel type d'algorithme faut-il mettre au point ? Le troisième sous-problème concerne la résolution du problème d'ordonnancement qui découle des deux sous-problèmes précédent. Nous proposons également dans ce travail une extension de la notation classique des problèmes d'ordonnancement au cas multicritère. Nous présentons ensuite les résultats de base de l'optimisation multicritère avant de détailler notre état de l'art sur les problèmes d'ordonnancement multicritères à une machine, à machines parallèles et de type flowshop.

Keywords: Scheduling, multicriteria optimization, state-of-the-art.

Received May, 1999.

${ }^{1}$ Laboratoire d'Informatique, École d'Ingénieurs en Informatique pour l'Industrie, 64 avenue Jean Portalis, 37200 Tours, France; e-mail: \{tkindt,billaut\}@e3i.univ-tours.fr

(c) EDP Sciences 2001 


\section{INTRODUCTION}

First researches on scheduling appeared in the 50's. From then on, problems became more and more complex because of the numerous practical constraints they wanted to take into account. Surprisingly the most important part of the literature on scheduling problems is dedicated to monocriterion problems whereas, in practice, the use of multiple criteria often enables to compute a more realistic solution for the decision maker. A survey on multicriteria one-machine scheduling problems can be found in $[27,35,49]$. They show that three kinds of problems have been tackled. The first one deals with problems in which a lexicographical order of criteria is minimized. The second class of problems considers a convex combination of criteria. The third class of problems is about the determination of all strict Pareto optima. Nagar et al. [64] present a similar survey on multimachine scheduling problems. Some results on the complexity of multicriteria scheduling problems are also presented in $[22,23,56]$. It appears that considering more than one criterion does not simplify the resolution of a scheduling problem. In terms of complexity, it means that multicriteria scheduling problems are at least as difficult as the corresponding monocriterion scheduling problems.

In this paper we try to highlight the interest of the multicriteria optimization theory to solve multicriteria scheduling problems. In Section 2 we propose a general framework for the resolution of multicriteria scheduling problems that allows to tackle multicriteria scheduling problems, according to Decision Aid concepts. After that, we present an extension to multicriteria problems of an existing notation for scheduling problems. Section 3 offers basic results on multicriteria optimization. Finally Section 4 is a brief survey on multicriteria scheduling problems as seen in the literature.

\section{FRAMEWORK AND NOTATION}

Minimizing several conflictuous criteria modifies the way scheduling problems are dealt with. In most cases a solution that optimally minimizes all the considered criteria does not exist. It implies that a new definition of optimality must be used, namely Pareto optimality definition. In fact two main definitions are encountered in multicriteria optimization literature: weak and strict Pareto optimality definitions.

Definition 1. Let us consider $K$ conflictuous criteria $Z_{i}$ to minimize. $\mathcal{S}$ is the set of solutions and $\mathcal{Z}$ its image in the criteria space. $x \in \mathcal{S}$ is a weak Pareto optimum (or a weak efficient solution) iff $\nexists y \in \mathcal{S}, x \neq y$, such that $Z_{i}(y)<$ $Z_{i}(x), \forall i=1 \ldots K . W E$ is the set of all weak Pareto optima and $Z_{W E}$ its image in the criteria space (i.e. the set of weakly non dominated criteria vectors).

Definition 2. $x \in \mathcal{S}$ is a strict Pareto optimum (or an efficient solution) iff $\nexists y \in \mathcal{S}, x \neq y$, such that $Z_{i}(y) \leq Z_{i}(x), \forall i=1 \ldots K$, with at least one strict inequality. $E$ is the set of all strict Pareto optima and $Z_{E}$ its image in the criteria space (i.e. the set of non dominated criteria vectors). One has $E \subseteq W E$. 
The decision maker is only interested in the strict Pareto optima, but unfortunately most available results compute a subset (or the entire set) of $W E$. A multicriteria scheduling problem consists in computing a feasible schedule that minimizes several criteria. This schedule is necessarily a strict Pareto optimum. The analyst is the person who designs an algorithm for the multicriteria scheduling problem under consideration. While dealing with such a problem, he has to solve three problems:

1. a modelization problem in which he interacts with the decision maker to define the relevant criteria, the set of considered solutions, etc. [75];

2. taking into account of the criteria problem, where he interacts with the decision maker to choose the resolution context (i.e. resolution method) and to define the shape of the objective function of the scheduling problem;

3. a scheduling problem for which he must determine a schedule that optimizes the determined objective function. The obtained solution is a strict Pareto optimum.

In the first problem the analyst interacts with the decision maker in order to obtain a model of the multicriteria problem under consideration. It means that for a multicriteria scheduling problem he has to define what kind of problem is tackled: a one machine problem, a flowshop problem, etc. Then he has to enumerate the particular constraints related to the problem: preemption, release dates, etc. Finally, in this problem he must also define the criteria to optimize, e.g. the makespan criterion, the total flowtime, etc.

In the second problem the analyst must determine the resolution context, i.e. the way to retrieve some information from the decision maker and to present him the computed Pareto optima. This is due to the fact that two strict Pareto optima can not be compared, so, in order to choose the final solution, the decision maker must help the resolution algorithm. Basically, Evans [33] distinguishes three kinds of resolution contexts. In the first one, the decision maker gives all the necessary information to the resolution algorithm (for instance the weights of criteria if a linear combination of criteria is minimized). This algorithm allows to compute a unique strict Pareto optimum. Such an algorithm is called an a priori algorithm. The algorithms of the second context are interactive ones that allow the decision maker to interactively determine his most preferred solution. In the third context, the resolution algorithm determines all the elements of the set $E$ and lets the decision maker choose the strict Pareto optimum he prefers. In this case, it is referred to as an a posteriori algorithm. After the resolution context is chosen, a multicriteria optimization result is used to build an objective function for the scheduling problem.

In the third problem the analyst has to propose an algorithm that solves the scheduling problem defined previously. The consequence is that we get a monocriterion sub-problem with additionnal parameters. It implies that this scheduling problem can have a non classical objective function. One can notice that if the 
criteria defined at the first step are the completion times of jobs, the optimization of a classical regular scheduling criterion (like makespan, total flowtime, etc.) allows to compute a Pareto optimum for these criteria.

The result obtained after the resolution of the three problems is an algorithm that is made up of two modules: the first one is the interface with the decision maker. This module sets the objective function parameters. The second module is dedicated to the resolution of the scheduling problem. For instance if a convex combination of criteria is minimized by an a posteriori algorithm, its first module computes all possible weights for the criteria, and then, for each value, it runs the second module that solves the corresponding scheduling problem.

Before presenting an overview of such results, we propose a notation for multicriteria scheduling problems based on the one presented in [14]. This notation is decomposed into three fields $\alpha|\beta| \gamma$ where the field $\gamma$ is dedicated to the criteria. The existing notations do not allow to take account of all possible ways to minimize some criteria. For multicriteria scheduling problems, we separate their notation into two levels. The first one refers to the general multicriteria scheduling problem and contains in the field $\gamma$ the list of minimized criteria. For instance, $F 2 \| C_{\max }, \bar{C}$ refers to the 2-machine flowshop problem where the aim is to minimize both $C_{\max }$ and $\bar{C}$ criteria.

The second level of the proposed extension refers to the scheduling problem for which the analyst proposes a resolution algorithm. In this case the different values we propose for the field $\gamma$ are:

- $Z$, if the aim is to minimize only criterion $Z$;

- $F_{\ell}\left(Z_{1}, \ldots, Z_{K}\right)$, if the aim is to minimize a linear combination of $K$ criteria;

- $P\left(Z_{1}, \ldots, Z_{K}\right)$ is a non decreasing function of the criteria. They are subjected to bound constraints (parametric approach);

- $\epsilon\left(Z_{u} / Z_{1}, \ldots, Z_{u-1}, Z_{u+1}, \ldots, Z_{K}\right)$, if the criterion to minimize is $Z_{u}$, and the other criteria are subjected to bound constraints ( $\epsilon$-constraint approach);

- $F_{T}\left(Z_{1}, \ldots, Z_{K}\right)$, if the aim is to minimize the distance to a known ideal solution using a Tchebycheff metric;

- $F_{T p}\left(Z_{1}, \ldots, Z_{K}\right)$, if the aim is to minimize the distance to a known ideal solution using a weighted Tchebycheff metric;

- $F_{T p a}\left(Z_{1}, \ldots, Z_{K}\right)$, if the aim is to minimize the distance to a known ideal solution using an augmented weighted Tchebycheff metric;

- $F_{s}\left(Z_{1}, \ldots, Z_{K}\right)$, if the aim is to maximize a special function that takes into account an ideal solution (goal-attainment approach);

- $\operatorname{Lex}\left(Z_{1}, \ldots, Z_{K}\right)$, if the aim is to minimize a lexicographical order of all criteria, i.e. to minimize criterion $Z_{1}$, then to minimize criterion $Z_{2}$ subject to the optimality of criterion $Z_{1}$, etc. The order in the Lex operator is the considered lexicographical order;

- \# $\left(Z_{1}, \ldots, Z_{K}\right)$, denotes the problem of enumeration of Pareto optima. Generally this approach is associated to an a posteriori resolution and consists in enumerating the set $E$. 
All these values refer to the different multicriteria optimization results presented in Section 3. The possible values for fields $\alpha$ and $\beta$ are the classical ones [14].

\section{AN OVERVIEW OF MULTICRITERIA OPTIMIZATION RESULTS}

Many results are available in multicriteria optimization literature. The aim of this section is to provide general guidelines on the most commonly used results. Steuer [86] presents a detailed review of some of these results (mainly for linear problems). Different approaches can be considered to take criteria into account, depending on different factors:

1. Tradeoff: are tradeoff between criteria allowed?

2. Weights: is it possible to associate a weight to each criterion?

3. Goal: is it possible to associate a particular goal value to each criterion?

4. Bound: does an upper bound exist for each criterion?

Depending on the approach, one factor or more can be taken into account. Several results are then available to compute Pareto optima. The different approaches generally transform the original multicriteria problem into a new monocriterion problem in which the objective function is an aggregation of the different criteria with new parameters.

- Geoffrion [43] proposes a result when a convex combination of criteria is minimized. This result allows to compute the set of proper Pareto optima (set PRE), that is a subset of $\mathrm{E}$.

Theorem 1. [43] Let $\mathcal{S}$ be the convex set of solutions and $K$ criteria $Z_{i}$ that are convex functions on $\mathcal{S}$. $x^{0}$ is a proper Pareto optimum iff $\exists \alpha \in \mathbb{R}^{K}$, with $\left.\alpha_{i} \in\right] 0 ; 1[$ and $\sum_{i=1}^{K} \alpha_{i}=1$ such that $x^{0}$ is an optimal solution of problem $\left(P_{\alpha}\right)$ :

Min $g(Z(x))$ with $g(Z(x))=\sum_{i=1}^{K} \alpha_{i} Z_{i}(x)$ subject to $x \in \mathcal{S}$

$P R E$ is the set of proper Pareto optima and one has PRE $\subseteq$ E.

For problems that can be modeled using a mixed integer or linear program with a finite number of constraints, one has $P R E=E$ [86]. If the weights $\alpha_{i}$ can be equal to 0 or 1 then the set $W E$ can be computed using Theorem 1. At last, if the convex hypotheses do not hold, then only the sufficient condition of the theorem is true. It means that every solution to problem $\left(P_{\alpha}\right)$ is a strict or weak Pareto optima (depending on $\alpha_{i}$ being equal to 0 and 1 or not). These computable solutions are called the supported Pareto optima and belong to the border of the convex hull of $\mathcal{Z}$. The other Pareto optima are non supported and belong to the inside of the convex hull of $\mathcal{Z}$ (see Fig. 1).

- The parametric approach consists in minimizing an objective function subject to bound constraints on the criteria.

Theorem 2. [85] Let $G_{Y}$ be the set of functions from $\mathbb{R}^{K}$ to $\mathbb{R}$ that are strictly increasing functions, lower bounded on $\mathcal{Z}$. Let $g \in G_{Y} . x^{0} \in \mathcal{S}$ is a strict Pareto 


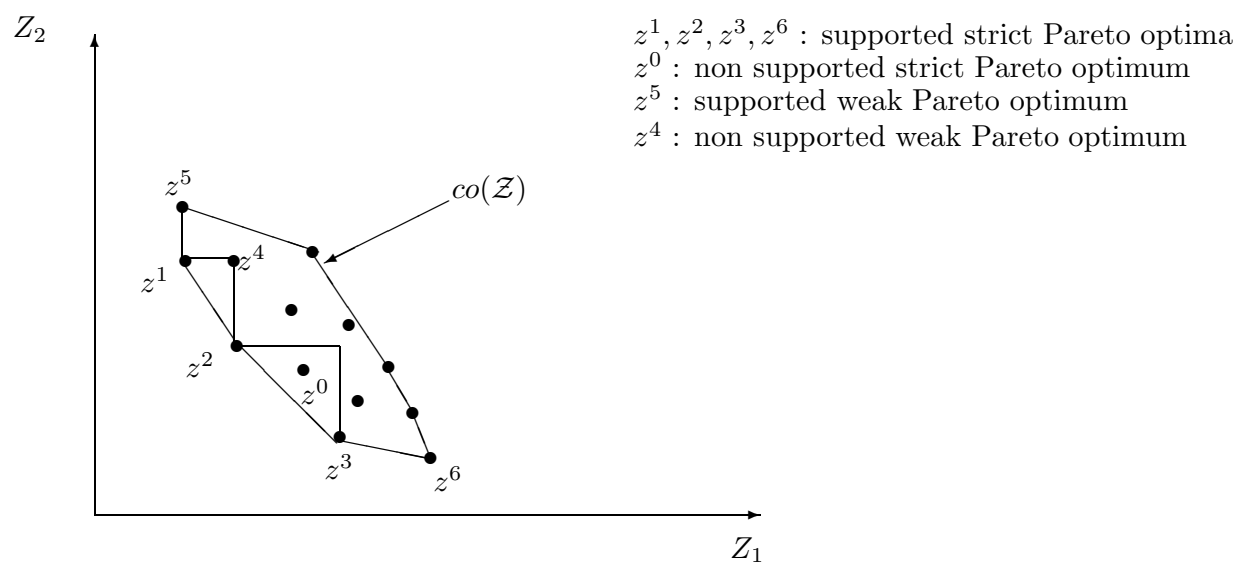

FiguRE 1. Supported and non supported Pareto optima.

optimum iff $\exists b \in \mathbb{R}^{K}$ such that $x^{0}$ is an optimal solution of problem $\left(P_{(g, b)}\right)$ :

$\operatorname{Min} g(Z(x))$

subject to $x \in \mathcal{S}$ and $Z(x) \leq b$.

The parametric approach is useful because Theorem 2 ensures that only the set $E$ can be computed by solving all possible problems $\left(P_{(g, b)}\right)$ when $g$ is fixed. A similar result holds for weak Pareto optima if one consider an increasing function instead of a strictly increasing function.

- A similar approach is the $\epsilon$-constraint approach and has first been proposed in [47]. A basic result is given in Theorem 3.

Theorem 3. [89] Let $k \in[1 ; K]$ be a number of criterion. If $\exists \epsilon^{k}=$ $\left(\epsilon_{1}^{k} ; \ldots ; \epsilon_{k-1}^{k} ; \epsilon_{k+1}^{k} ; \ldots ; \epsilon_{K}^{k}\right) \in \mathbb{R}^{K-1}$ such that $x^{0} \in \mathcal{S}$ is an optimal solution of problem $\left(P_{\epsilon^{k}}\right)$ :

$\operatorname{Min} Z_{k}(x)$

subject to $x \in \mathcal{S}$ and $Z_{i}(x) \leq \epsilon_{i}^{k} \forall i \in[1 ; K], i \neq k$

then $x^{0}$ is a weak Pareto optimum.

It can be shown that the set of all solutions determined using Theorem 3 is a subset of $W E$ and that this subset contains set $E$. A more general form of this theorem considers all problems $\left(P_{\epsilon^{k}}\right), \forall k=1 \ldots K, \forall \epsilon^{k}$. It states that the set of all solutions to all problems $\left(P_{\epsilon^{k}}\right)$ is a subset of $W E[62]$. If the convex hypothesis for $\mathcal{S}$ and $Z_{i}, \forall i=1 \ldots K$, holds, then the whole set $W E$ can be determined by the general form of Theorem 3 .

- An other well known way to compute Pareto optima consists in minimizing a metric over the set $\mathcal{Z}$. These approaches are generally based on an utopian point $z^{u t}$ defined by $z_{i}^{u t} \leq \min _{x \in \mathcal{S}}\left(Z_{i}(x)\right), \forall i=1 \ldots K$, with at least one strict inequality. Here, we consider the augmented weighted Tchebycheff metric. 
Theorem 4 (see for instance [89]). $x^{0} \in \mathcal{S}$ is a strict Pareto optimum iff $\exists \lambda \in$ $\mathbb{R}_{+*}^{K}, z^{u t} \in \mathbb{R}^{K}$ an utopian point and $\exists \rho \in \mathbb{R}_{+}^{*}$ a small value, such that $x^{0}$ is an optimal solution of problem $\left(P_{(\lambda, \rho)}\right)$ :

$\operatorname{Min}\left\|Z(x)-z^{u t}\right\|_{T p a}$

subject to $x \in \mathcal{S}$

with $\left\|Z(x)-z^{u t}\right\|_{T p a}=\max _{1 \leq i \leq K}\left(\lambda_{i}\left|Z_{i}(x)-z_{i}^{u t}\right|\right)+\rho \sum_{i=1}^{K}\left|Z_{i}(x)-z_{i}^{u t}\right|$.

The main difficulty in the use of Theorem 4 lies in the setting of value $\rho$. For instance, Steuer [86] proposes to set this value around $10^{-6}$. Some particular cases are the weighted Tchebycheff metric $(\rho=0)$ and the Tchebycheff metric $(\rho=0$ and $\left.\lambda_{i}=1, \forall i=1 \ldots K\right)$ for which Bowman [16] proposes some results, yet weaker than that of Theorem 4 . Other metrics such as the weighted $L_{p}$ metric can be used.

- The goal-attainment approach has been proposed by Gembicki [42].

Theorem 5. $[42,103] x^{0} \in \mathcal{S}$ is a weak Pareto optimum iff $\exists z^{\text {ref }} \in \mathbb{R}^{K}$ a reference point and $w \in \mathbb{R}_{+*}^{K}$ a weight vector such that $x^{0}$ is an optimal solution of problem $\left(P_{\left(z^{\text {ref }}, w\right)}\right)$ :

$$
\operatorname{Max} g(Z(x)) \text { with } g(Z(x))=\min _{1 \leq i \leq K}\left(\frac{1}{w_{i}}\left(z_{i}^{\text {ref }}-Z_{i}(x)\right)\right.
$$

subject to $x \in \mathcal{S}$.

The advantage of this theorem compared to Theorem 4 lies in the reference criteria vector: in the goal-attainment approach the reference point can be any point in the criteria space. One disadvantage is that for fixed weights $w_{i}$, the computed weak Pareto optimum depends on the relative position of the goal $z^{\text {ref }}$ compared to the set $\mathcal{Z}$ (see [89]). On the other hand, one can show that an optimal solution to the problem $\operatorname{Lex}\left(\min _{1 \leq i \leq K}\left(\frac{1}{w_{i}}\left(z_{i}^{\text {ref }}-Z_{i}(x)\right)\right), \sum_{i=1}^{K}\left(\frac{1}{w_{i}}\left(z_{i}^{\text {ref }}-Z_{i}(x)\right)\right)\right)$ is a strict Pareto optimum [90].

- Other approaches are possible to minimize multiple criteria. One of the most used in scheduling problems is the minimization of a lexicographical order of criteria, for instance $Z_{1} \rightarrow Z_{2} \rightarrow \ldots \rightarrow Z_{K}$. The aim is to find a solution $x^{0} \in S^{K}$ with $S^{i}=\left\{x \in \mathcal{S}^{i-1} / Z_{i}(x)=\min _{x^{\prime} \in \mathcal{S}^{i-1}}\left(Z_{i}\left(x^{\prime}\right)\right)\right\}, \forall i=1 \ldots K$, and $S^{0}=\mathcal{S}$. A solution to this problem is a strict Pareto optimum and each solution $x^{1} \in S^{i}, \forall i=1 \ldots K$, is a weak Pareto optimum. Notice that with this approach no tradeoff between criteria is allowed, so it can only be used in an a priori resolution algorithm. At last an obvious way to solve a multicriteria problem is to propose an algorithm that enumerates the whole set $E$. Other particular approaches can be found in [29].

\section{AN OVERVIEW OF MULTICRITERIA SCHEDULING PROBLEMS}

Surveys on multicriteria scheduling literature only focus on solved scheduling problems, but do not present the associated multicriteria optimization results. For some multicriteria scheduling problems, authors choose an objective function that 
is not relevant, regarding the scheduling problem they consider. The knowledge of some basic results in the field of multicriteria optimization can prevent such mistakes. For instance, an optimal solution to the problem of minimizing a convex combination of criteria is a supported Pareto optimum for non convex problems. It means that some solutions (the non supported Pareto optima) that are potentially interesting for the decision maker can never be determined with such an approach. On another hand, the minimization of a criterion subjected to bound constraints on the other criteria ( $\epsilon$-constraint approach) leads to a weak Pareto optimum and not necessarily to a strict Pareto optimum. Unfortunately, we note that most of multicriteria scheduling problems tackled in the literature deal with one of these two ways to handle the criteria. It implies that either some interesting solutions can not be obtained, or some non interesting solutions are determined. The knowledge of these theoretical results from the multicriteria optimization field, would have allowed to avoid these problems. The notations used in this section are described in Table 1.

TABLE 1. Basic notations in scheduling.

\begin{tabular}{|c|c|c|}
\hline Data & $\begin{array}{l}n \\
p_{i}\left(p_{i, j}\right) \\
d_{i} \\
s_{i} \\
r_{i}\end{array}$ & $\begin{array}{l}\text { number of jobs } \\
\text { processing time of job } i \text { (on machine } j \text { ) } \\
\text { due date of job } i \\
\text { desired starting time of job } i \\
\text { release date of job } i\end{array}$ \\
\hline Variables & $\begin{array}{l}C_{i} \\
E_{i} \\
L_{i} \\
T_{i} \\
U_{i}\end{array}$ & $\begin{array}{l}\text { completion time of job } i \\
\text { earliness of job } i: E_{i}=\max \left(0, d_{i}-C_{i}\right) \\
\text { lateness of job } i: L_{i}=C_{i}-d_{i} \\
\text { tardiness of job } i: T_{i}=\max \left(0, C_{i}-d_{i}\right) \\
\text { flag of tardiness for job } i: U_{i}=1 \text { if } i \text { is tardy and } 0 \text { otherwise }\end{array}$ \\
\hline Constraints & $\begin{array}{l}\text { permu } \\
\text { pmtn } \\
\text { nmit } \\
S_{s d} \\
\end{array}$ & $\begin{array}{l}\text { in a flowshop problem the job sequence is the same for each machine } \\
\text { jobs can be interrupted and resumed later } \\
\text { no machine idle times are allowed } \\
\text { sequence dependent setup times occur between jobs }\end{array}$ \\
\hline Criteria & $\begin{array}{l}f_{\max } \\
C_{\max } \\
L_{\max } \\
L_{\min } \\
T_{\max } \\
E_{\max } \\
\bar{C}\left(\bar{C}^{w}\right) \\
\bar{T}\left(\bar{T}^{w}\right) \\
\bar{E}\left(\bar{E}^{w}\right) \\
\bar{U}\left(\bar{U}^{w}\right)\end{array}$ & $\begin{array}{l}\text { general maximum function strictly increasing with the completion times } \\
\text { maximum completion of jobs: } C_{\max }=\max _{i=1 . . n}\left(C_{i}\right) \\
\text { maximum lateness of jobs: } L_{\max }=\max _{i=1 . . n}\left(L_{i}\right) \\
\text { minimum lateness of jobs: } L_{\min }=\min _{i=1 . . n}\left(L_{i}\right) \\
\text { maximum tardiness of jobs: } T_{\max }=\max _{i=1 . . n}\left(T_{i}\right) \\
\text { maximum earliness of jobs: } E_{\max }=\max _{i=1 . . n}\left(E_{i}\right) \\
\text { Sum of completion times: } \bar{C}=\sum_{i=1}^{n}\left(C_{i}\right) \text { (weighted sum) } \\
\text { Sum of tardiness: } \bar{T}=\sum_{i=1}^{n}\left(T_{i}\right) \text { (weighted sum) } \\
\text { Sum of earliness: } \bar{E}=\sum_{i=1}^{n}\left(E_{i}\right) \text { (weighted sum) } \\
\text { Number of late jobs: } \bar{U}=\sum_{i=1}^{n}\left(U_{i}\right) \text { (weighted sum) }\end{array}$ \\
\hline
\end{tabular}

One class of criteria considered in scheduling literature contains the criteria based on completion times. The makespan, noted $C_{\max }$, is the length of the 
scheduling period that must be minimized. The total flowtime criterion can also be minimized. This criterion, noted $\bar{C}$, is the sum of the completion times of jobs. It can either be weighted or not. A second class of well known criteria contains the criteria based on due dates. Maximum cost functions are for example the maximum lateness of jobs (criterion $L_{\max }$ ) or the maximum tardiness of jobs (criterion $T_{\max }$ ). Another possibility is to minimize the maximum earliness of jobs (criterion $E_{\max }$ ). Sum cost functions are for example the mean earliness (criterion $\bar{E})$, the mean tardiness (criterion $\bar{T}$ ) or the number of late jobs (criterion $\bar{U}$ ). All these sum criteria can also be considered with weighted jobs.

Most multicriteria scheduling problems are related to only one machine problems, and the most frequent criteria to just-in-time problems. In this section we present an overview of one machine, parallel machines and flowshop multicriteria scheduling problems.

The survey is organized as follows: for each problem, we present the literature according to a classification based on the kind of the criteria optimized. The first class contains problems with at least one criterion based on the sum of completion times of jobs. The second class contains problems with at least one criterion being a crashing time cost of jobs. The third class concerns multicriteria problems with a tool changing cost. The two last classes contain problems with only due date based criteria. We distinguish problems dealing with Just-In-Time scheduling from problems with other due date based criteria. A detailed version of this survey is proposed in [89].

\subsection{ONE MACHINE SCHEDULING PROBLEMS}

Literature on multicriteria scheduling mainly deals with one machine scheduling problems. They are of some interest since their properties can be either generalized to more complicated environments or used to design some heuristic algorithms for multi-machine problems.

A general result is given by Hoogeveen [49] who shows the problem $1 \| \epsilon$ $\left(f_{\max }^{1} / f_{\max }^{2}, \ldots, f_{\max }^{K}\right)$ is polynomially solvable when $f_{\max }^{i}(S)=$ $\max _{1 \leq j \leq n}\left(f_{j}^{i}\left(C_{j}(S)\right)\right)$ with $f_{j}^{i}$ increasing functions of the completion times $C_{j}$. The author shows that the cardinality of set $E$ is at most $(n(n-1) / 2+1)^{K-1}$.

\subsubsection{Minimizing the sum of completion times of jobs}

In this section we present the problems in which the mean completion time of jobs is considered as one of the criteria.

The problem $1\left|d_{i}\right| \epsilon\left(\bar{C} / L_{\max }\right)$ with $L_{\max }=0$ has been solved by Smith [84]. When $L_{\max }$ criterion has no mandatory value, the problem is polynomially solvable and has been tackled by Heck and Roberts [48]. Van Wassenhove and Gelders [97] and Nelson et al. [66] propose algorithms to compute the set $E$. Unfortunately, the branch-and-bound algorithm of Nelson et al. actually computes a subset of the set $W E$. The problem $1\left|d_{i}\right| F_{\ell}\left(T_{\max }, \bar{C}\right)$ belongs to the class $\mathcal{P}$ and has been studied by Sen and Gupta [79]. Their algorithm computes supported Pareto optima. Some problems with a general maximum cost function $f_{\max }$ are considered. 
Emmons [30] studies the problem 1\|Lex $\left(f_{\max }, \bar{C}\right)$ that belongs to the class $\mathcal{P}$. John [51] proposes an enumerative algorithm that computes the set $E$. The author shows that $|E|<\frac{1}{4} n^{2}\left(p_{\max }-p_{\min }\right)$ where $p_{\max }$ and $p_{\min }$ are the maximum and the minimum processing time of jobs. Hoogeveen and Van de Velde [50] propose an algorithm that also computes the set $E$. It proceeds by iteratively solving the problem $1 \| \epsilon\left(\bar{C} / f_{\max }\right)$. The authors show that $|E|<n(n-1) / 2+1$. The problem $1\left|d_{i}, n \operatorname{mit}\right| \epsilon\left(\bar{C} / E_{\max }\right)$ for which the insertion of idle times before jobs is not allowed is strongly $\mathcal{N} \mathcal{P}$-hard. Azizoglu et al. [5] propose a heuristic that approximates a subset of $W E$. The problem $1\left|d_{i}, n m i t\right| F_{\ell}\left(\bar{C}, L_{\max }-L_{\min }\right)$ for which the complexity is unknown has been tackled by Sen et al. [80]. They propose a branch-and-bound algorithm that enumerates the set of supported strict Pareto optima. The problem $1\left|d_{i}\right| \operatorname{Lex}(\bar{U}, \bar{C})$ is $\mathcal{N} \mathcal{P}$-hard. Emmons [31] proposes a heuristic to solve it. For the problem $1\left|d_{i}\right| \epsilon(\bar{C} / \bar{U})$ Nelson et al. [66] propose a branch-and-bound algorithm and two heuristics. These algorithms compute or approximate a subset of set $W E$. The problem $1\left|d_{i}\right| \#(\bar{T}, \bar{C})$ has been solved by Lin [60] who proposes a dynamic programming algorithm. This problem is $\mathcal{N} \mathcal{P}$ hard. The problem $1\left|d_{i}\right| F_{\ell}(\bar{E}, \bar{C})$ has been tackled by Fry and Leong [38]. Its complexity is unknown and a mixed integer program is proposed by the authors. The problem $1\left|d_{i}\right| \epsilon\left(\bar{C} / \bar{U}, T_{\max }\right)$ has been studied by Nelson et al. [66]. They propose a branch-and-bound algorithm that computes a subset of set $W E$.

\subsubsection{Minimizing the total weighted completion time of jobs}

Other works deal with the minimization of the total weighted completion time of jobs. Chen and Bulfin [21] solve fifteen polynomial problems with unit processing time jobs. The problem $1\left|d_{i}\right| \operatorname{Lex}\left(L_{\max }, \bar{C}^{w}\right)$ has been shown strongly $\mathcal{N} \mathcal{P}$-hard by Hoogeveen [49]. For this problem, a dynamic programming algorithm is proposed by Chand and Schneeberger [18]. The problem $1\left|d_{i}\right| \epsilon\left(\bar{C}^{w} / L_{\max }\right)$ with $L_{\max }=0$ is strongly $\mathcal{N} \mathcal{P}$-hard and is studied in $[10,19,30,84]$. Several heuristics are proposed by Heck and Roberts [48] and Burns [17] when no value on $L_{\max }$ criterion is imposed. The equivalence between the problem with a fixed value for $L_{\max }$ and that without a fixed value for $L_{\max }$ has been shown by Miyazaki [63]. Moreover the author proposes an efficient algorithm to solve this problem. The problem $1\left|d_{i}\right| F_{\ell}\left(\bar{C}^{w}, \bar{T}^{w}\right)$ is strongly $\mathcal{N} \mathcal{P}$-hard and has been studied by Van Wassenhove and Gelders [96]. The authors propose three branch-and-bound procedures and a dynamic programming algorithm to solve it.

\subsubsection{Minimizing the total weighted crashing time cost of jobs}

When the exact processing times of jobs are variables to be computed, the scheduling problem can be modeled as a multicriteria scheduling problem. In this case, one has to minimize "crashing costs" and we have $p_{i} \in\left[p_{i} ; \bar{p}_{i}\right]$. This kind of problem can occur in hoist scheduling problems in which materials (jobs) must be plundged in a chemical bath. Chemical engineers are able to define a lower bound $\underline{p}_{i}$ and an upper bound $\bar{p}_{i}$ on the duration $p_{i}$ of the processing. The aim is to compute the exact processing time for each job. Another important constraint is 
linked to the transportation system between baths. If there is only one bath, the problem is a one machine scheduling problem and the constraint on the transportation system disappears. The problem $1\left|p_{i} \in\left[\underline{p}_{i} ; \bar{p}_{i}\right], d_{i}\right| F_{\ell}\left(T_{\max }, \overline{C C}^{w}\right)$ is tackled by Vickson [99]. The criterion $\overline{C C}^{w}$ is the weighted sum of the crashing costs of jobs. This problem belongs to the class $\mathcal{P}$. The problem $1\left|p_{i} \in\left[p_{i} ; \bar{p}_{i}\right], d_{i}\right| \epsilon\left(\overline{C C}^{w} / T_{\max }\right)$ is solved by Van Wassenhove and Baker [95]. The authors show that their polynomial algorithm only computes the set of supported strict Pareto optima. This algorithm is then extended to solve problems with a general maximum cost function $f_{\max }$ instead of criterion $T_{\max }$. Some additional assumptions hold for the function $f_{\max }$. The problem $1\left|p_{i} \in\left[\underline{p}_{i} ; \bar{p}_{i}\right]\right| F_{\ell}\left(\bar{C}, \overline{C C}^{w}\right)$ is also solved by Vickson [99]. This problem belongs to class $\mathcal{P}$. When the weighted mean completion time criterion is considered, the problem remains open. Vickson [98] conjectures that the problem is $\mathcal{N} \mathcal{P}$-hard and presents a heuristic and a branch-and-bound algorithm.

\subsubsection{Minimizing the tool changing costs}

Some multicriteria scheduling problems deal with tool changing costs. The problem $1 \mid$ class, order, $S_{s d} \mid \operatorname{Lex}(\overline{S C}, \overline{A C})$ has been tackled by Gupta et al. [44]. The jobs belong to different classes and different orders have to be processed. Each order is made of different jobs. Sequence dependent setup times occur when jobs from different classes are processed in sequence. The aim is to minimize the sum of the setup costs $\overline{S C}$ and the carrying costs $\overline{A C}$. The authors propose an $O(n \log (m))$ polynomial time algorithm to solve it. An $O(n)$ algorithm for problem $1 \mid$ class, order, $S_{s d} \mid \operatorname{Lex}(\overline{A C}, \overline{S C})$ is also presented. In [15] two $\mathcal{N} \mathcal{P}$-hard problems are tackled. Bourgade et al. are interested in the problem $1\left|d_{i}, S_{s d}\right| F\left(\overline{S C}, T_{\max }\right)$ with two different forms of function $F$. The problem $1\left|S_{s d}\right| F_{\ell}\left(\overline{S C}, \bar{C}^{w}\right)$ is $\mathcal{N} \mathcal{P}$ hard. Barnes and Vanston [11] propose a heuristic, a branch-and-bound algorithm and a dynamic programming algorithm.

\subsubsection{Minimizing due date based criteria}

Chen and Bulfin [21] solve problems with due date based criteria. Eighteen polynomial problems with unit processing times are considered. In these problems either a lexicographical order of criteria or a convex combination of criteria is minimized. The problem $1\left|d_{i}\right| \operatorname{Lex}\left(\bar{U}, T_{\max }\right)$ is studied by Shantikumar [82]. It is an open problem. A branch-and-bound algorithm is proposed. The problem $1\left|d_{i}\right| \epsilon\left(T_{\max } / \bar{U}\right)$ has been tackled by Nelson et al. [66]. They present a branchand-bound algorithm that computes a subset of the set $W E$. They also present a heuristic that approximates this subset.

\subsubsection{Minimizing Just-In-Time criteria}

Just-In-Time (JIT) scheduling problems have often been studied. They belong to the class of multicriteria scheduling problems since they involve measuring earliness and tardiness of jobs. Such problems frequently arise in industry, 
as for instance in car seat production. When a semi-finished car is stored, an order is sent to the car seat maker who has five hours to produce and send the desired seats. Finishing too early induces high storage costs for the car maker. Finishing too late implies that some cars are re-processed from previous part in the assembly line. For these reasons, the car seat must be delivered on time. JIT problems are the most important part of the literature on multicriteria scheduling problems. Hoogeveen [49] is interested in problems $1 \mid s_{i}, d_{i}, d_{i}-s_{i} \leq p_{i}, n$ mit $\mid \epsilon\left(L_{\max } / P_{\max }\right)$ and $1\left|s_{i}, d_{i}, d_{i}-s_{i} \leq p_{i}\right| \epsilon\left(L_{\max } / P_{\max }\right)$. $P_{\max }$ is the maximum promptness and is defined by $P_{\max }=\max _{1 \leq i \leq n}\left(s_{i}-t_{i}\right)$ where $t_{i}$ is the real starting time of job $J_{i}$. The author shows that these problems are polynomially solvable. When the constraint $d_{i}-s_{i} \leq p_{i}$ does not hold, these problems are strongly $\mathcal{N} \mathcal{P}$-hard. An $O(n \log (n))$ algorithm is proposed for problem $1\left|s_{i}, d_{i}, d_{i}-s_{i} \leq p_{i}, n m i t\right| \epsilon\left(L_{\max } / P_{\max }\right)$. Then an algorithm that computes the set $E$ is introduced and the author shows that the cardinality of this set is at most $n$. For the problem $1\left|s_{i}, d_{i}, d_{i}-s_{i} \leq p_{i}\right| \epsilon\left(L_{\max } / P_{\max }\right)$ an $O\left(n^{2} \log (n)\right)$ algorithm is proposed. The problem $1\left|d_{i}\right| F_{\ell}(\bar{E}, \bar{T})$ with $F_{\ell}(\bar{E}, \bar{T})=\bar{E}+\bar{T}$ is $\mathcal{N} \mathcal{P}$ hard. Garey et al. [41] propose an $O(n \log (n))$ algorithm to compute the optimal starting times of jobs when the sequence of jobs is fixed. Moreover they show how this algorithm can be used to solve problems with additionnal constraints. The problem $1 \mid d_{i}=d \geq \sum_{i=1}^{n} p_{i}, n$ mit $\mid F_{\ell}(\bar{E}, \bar{T})$ with $F_{\ell}(\bar{E}, \bar{T})=\bar{E}+\bar{T}$ has been studied by Kanet [52] who proposes an $O\left(n^{2}\right)$ algorithm. The problem $1 \mid d_{i}=d \geq$ $\delta$, nmit $\mid F_{\ell}(\bar{E}, \bar{T})$ with $F_{\ell}(\bar{E}, \bar{T})=\bar{E}+\bar{T}$ has been tackled by Bagchi et al. [8]. They consider the case where $d \geq \delta$ with either $\delta=p_{1}+p_{3}+\ldots+p_{n}$ if $n$ is odd or $\delta=p_{2}+p_{4}+\ldots+p_{n}$ if $n$ is even. They propose a polynomial time algorithm to solve it. Bector et al. [12] deal with the problem $1 \mid d_{i}=d$, d unknown, nmit $\mid F_{\ell}(\bar{E}, \bar{T})$ with $F_{\ell}(\bar{E}, \bar{T})=\bar{E}+\bar{T}$. They propose a mixed integer program for this problem and a polynomial algorithm. For the problems $1\left|p_{i}=1, d_{i}, n m i t\right| \epsilon(\bar{E} / \bar{U}), 1 \mid p_{i}$ $=1, d_{i}, n$ mit $\mid \epsilon\left(E_{\max } / \bar{U}\right)$ and $1\left|p_{i}=1, d_{i}, n \operatorname{mit}\right| \epsilon\left(F_{\ell}(\bar{E}, \bar{T}) / \bar{U}\right)$ with $F_{\ell}(\bar{E}, \bar{T})=$ $\bar{E}+\bar{T}$, Kondakci et al. [53] propose some linear programs. Then, for all these problems, they propose algorithms to compute the set $E$. The problem $1 \mid d_{i}=d \geq$ $\sum_{i=1}^{n} p_{i}, n m i t \mid F_{\ell}\left(\bar{E}^{w}, \bar{T}^{w}\right)$ with $F_{\ell}\left(\bar{E}^{w}, \bar{T}^{w}\right)=\sum_{i=1}^{n} p_{i}\left(E_{i}+T_{i}\right)$ has been tackled by Ahmed and Sundararaghavan [2] who propose an optimal algorithm. The problem $1\left|p_{i}=1, d_{i}\right| F\left(E_{i}, T_{i}\right)$ with $F\left(E_{i}, T_{i}\right)=\max _{1 \leq i \leq n}\left(E_{i}+T_{i}\right)$ has been studied by Garey et al. [41]. They propose an $O(n \log (n))$ algorithm to solve it. When the earliness of jobs are related to desired starting times $s_{i}$ of jobs, Sidney [83] deals with the problem $1 \mid$ agree $s_{i}, d_{i} \mid F\left(f\left(T_{\max }\right), g\left(E_{\max }\right)\right)$ where $F\left(f\left(T_{\max }\right), g\left(E_{\max }\right)\right)=$ $\max \left(f\left(T_{\max }\right), g\left(E_{\max }\right)\right)$ and agree $s_{i}$ means that starting times are agreeable, i.e. $s_{i} \leq s_{j} \Leftrightarrow d_{i} \leq d_{j}, \forall i, j$. This problem belongs to the class $\mathcal{P}$ and Sidney proposes an $O\left(n^{2}\right)$ algorithm. In [55] an improved algorithm in $O(n \log (n))$ is presented. For the problem $1 \mid d_{i}$ unknown, nmit, $A \mid F_{\ell}(\bar{E}, \bar{T}, \bar{A})$ where criterion $\bar{A}$ is the total deviation of computed $d_{i}$ from the desired common due date $A$, Seidmann et al. [77] propose an $O(n \log (n))$ algorithm to solve it. The problem $1 \mid d_{i}=d$, d unknown, $n m i t \mid F_{\ell}(\bar{E}, \bar{T}, d)$ has also been tackled by Seidmann et al. [70]. They propose a polynomial algorithm. They also study the extension of this algorithm to similar 
problems. The problem $1 \mid d_{i}=d$, d unknown, lots, nmit $\mid F_{\ell}(\bar{E}, \bar{T}, \bar{B}, d)$ where criterion $\bar{B}$ is the number of lots completed after date $d$ is studied by Chen [24]. In this problem the jobs can be grouped in lots. The author proposes a dynamic programming algorithm which complexity is $O\left(n^{5}\right)$. Fry and Leong [37] propose a mixed integer model for the $\mathcal{N} \mathcal{P}$-hard problem $1\left|d_{i}\right| F_{\ell}(\bar{E}, \bar{T})$. For the problem $1\left|d_{i}=d, t_{0}=0, n m i t\right| F_{\ell}(\bar{E}, \bar{T})$ with $t_{0}$ the starting time of the schedule, Sundararaghavan and Ahmed [87] propose a heuristic. Bagchi et al. [7] propose a branch-and-bound algorithm to solve the problem $1 \mid d_{i}=d<\sum_{i=1}^{n} p_{i}$, $n$ mit $\mid F_{\ell}(\bar{E}, \bar{T})$ which is $\mathcal{N} \mathcal{P}$-hard. The problem $1\left|d_{i}=d<\delta, n m i t\right| F_{\ell}(\bar{E}, \bar{T})$ has been studied by Bagchi et al. [8]. When $d<\delta$, with eiter $\delta=p_{1}+p_{3}+\ldots+p_{n}$ if $n$ is odd or $\delta=p_{2}+p_{4}+\ldots+p_{n}$ if $n$ is even, the authors propose a branch-and-bound algorithm. Szwarc [88] also studies this problem when $t_{0}=0$. He proposes a branchand-bound algorithm. The problem $1\left|s_{i}, d_{i}\right| F_{\ell}(\bar{E}, \bar{T})$ with $F_{\ell}(\bar{E}, \bar{T})=\bar{E}+\bar{T}$ has been tackled by Koulamas [54]. The earliness of each job is specifically defined by $E_{i}=\max \left(s_{i}-t_{i}, 0\right)$ with $t_{i}$ its real starting time. The author shows that this problem is $\mathcal{N} \mathcal{P}$-hard and he presents seven heuristics and an optimal algorithm. Chand and Schneeberger [20] deal with the problem $1 \mid d_{i}$, nmit $\mid \epsilon\left(\bar{E}^{w} / \bar{U}\right)$. They show that this problem is $\mathcal{N} \mathcal{P}$-hard and propose a heuristic and a dynamic programming algorithm. The problem $1\left|d_{i}\right| F_{\ell}\left(\bar{E}^{w}, \bar{T}^{w}\right)$ is strongly $\mathcal{N} \mathcal{P}_{-}$ hard and has been studied by Fry et al. [34] who propose a heuristic using linear programing to solve it. Fry and Blackstone [36] present a mixed integer program for this problem. When no machine idle time is allowed, Ow and Morton $[68,69]$ propose several filtered beam search algorithms. The problem $1 \mid d_{i}=$ $d \geq \sum_{i=1}^{n} p_{i}$, nmit $\mid F_{\ell}\left(\bar{E}^{w}, \bar{T}^{w}\right)$ is $\mathcal{N} \mathcal{P}$-hard. Van den Akker et al. [94] propose an algorithm combining Lagrangean relaxation and column generation. Azizoglu and Webster [6] consider the problem in which there exist classes of jobs and setup times between jobs of different classes. The problem tackled is noted $1 \mid d_{i}=d \geq$ $\sum_{i=1}^{n} p_{i}, S_{s d}, n m i t \mid F_{\ell}\left(\bar{E}^{w}, \bar{T}^{w}\right)$. They propose some dominance conditions and a branch-and-bound algorithm. A filtered beam search based heuristic is also presented. The problem $1 \mid d_{i}=d$, d unknown, $S_{s d}, n m i t \mid F_{\ell}\left(\bar{E}^{w}, \bar{T}^{w}\right)$ has been studied by Webster et al. [102]. It is a $\mathcal{N} \mathcal{P}$-hard problem and the authors propose a genetic algorithm to solve it. The problem $1\left|d_{i}\right| F_{\ell}(\bar{C}, \bar{T}, \bar{E})$ is $\mathcal{N} \mathcal{P}$-hard. Fry et al. [39] propose a branch-and-bound algorithm to solve it. The problem $1\left|d_{i}, n m i t\right| F\left(E_{i}, T_{i}\right)$ with $F\left(E_{i}, T_{i}\right)=\sum_{i=1}^{n}\left(E_{i}+T_{i}\right)^{2}$ has been tackled by Gupta and Sen [46]. Its complexity is unknown. They propose a branch and bound algorithm and a heuristic. The problem $1 \mid d_{i}=d$, d unknown, nmit $\mid F\left(E_{i}, T_{i}\right)$ has been studied by Bagchi et al. [9]. It remains open. The problem $1 \mid d_{i}$ unknown, nmit $\mid F_{\ell}\left(\bar{E}^{w}, \bar{T}^{w}\right)$ has been studied by Adamopoulos and Pappis [1]. Its complexity is open. For problem $1\left|d_{i}=d, n m i t\right| F_{\ell}\left(\sum_{i=1}^{n} E_{i}^{2}, \sum_{i=1}^{n} T_{i}^{2}\right)$, Bagchi et al. [7] propose a branch and bound algorithm. Its complexity is open. The problem $1\left|d_{i}\right| F(\bar{E}, \bar{T}, \bar{C})$ with $F(\bar{E}, \bar{T}, \bar{C})=(1-\alpha)(\bar{E}-\bar{T})^{2}+\alpha \bar{C}$ is open. Dileepan and Sen [28] present a branch and bound algorithm to solve it. 


\subsection{PARAllel MAChines SChEDUling PROBlems}

These problems are important because in practice there are often multiple resources dedicated to the processing of some operations (in industry as well as in parallel computing). Moreover resolution algorithms for such problems can be used in more general decomposition algorithms for multi-stage shop problems. These heuristics can be efficient if the multiple resources stage is a bottleneck one. Few multicriteria scheduling problems on parallel machines have been dealt with in the literature.

\subsubsection{Minimizing completion times of jobs}

The problem $P|p m t n| \operatorname{Lex}\left(\bar{C}, C_{\max }\right)$ is polynomially solvable. Leung and Young [57] present an $O(n \log (n))$ algorithm to solve it. The problem $Q|p m t n|$ $\epsilon\left(\bar{C} / C_{\max }\right)$ has been considered by McCormick and Pinedo [61]. The preemption of jobs occur at real times. They propose an algorithm that computes all the strict Pareto optima that correspond to extreme points of the solution set, which is a polytope in the decision space. The complexity of this algorithm is in $O\left(m^{3} n\right)$. The problem $P\left|p m t n, d_{i}\right| \operatorname{Lex}\left(C_{\max }, L_{\max }\right)$ is polynomially solvable. T'kindt et al. [91] propose a pseudo-polynomial time implementation in $O\left(n^{4} \log _{2}\left(C_{\max }^{*}\right)\right)$ using Horn's procedure for the $P\left|p m t n, \widetilde{d}_{i}\right|-$. The problem $P \mid$ pmtn,$d_{i} \mid \operatorname{Lex}\left(L_{\max }^{w}, \bar{C}^{w}\right)$ with $L_{\max }^{w}=\max _{i=1}\left(w_{i} L_{i}\right)$ has been studied by T'kindt et al. [90]. This problem is strongly $\mathcal{N} \mathcal{P}$-hard and the authors propose a heuristic to solve it.

\subsubsection{Maximizing the total production margin of jobs}

T'kindt et al. [92] consider the problem $R|p m t n| F_{\ell}\left(I_{\max }, \bar{M}\right)$ where the preemption of jobs occurs at real times. The aim is to maximize the total margin $\bar{M}$ and to minimize the maximum difference of machines workload $I_{\max }$. The authors propose an interactive algorithm to solve the problem. In [3] a similar algorithm is presented when the set of solutions is a discrete set.

\subsubsection{Minimizing the total weighted crashing time cost of jobs}

When crashing costs are involved Alidaee and Ahmadian [4] study the problems $R\left|p_{i, j} \in\left[\underline{p}_{i, j} ; \bar{p}_{i, j}\right]\right| F_{\ell}\left(\bar{C}, \overline{C C}^{w}\right)$ and $R \mid p_{i, j} \in\left[\underline{p}_{i, j} ; \bar{p}_{i, j}\right], d_{i}=d$, d unknown $\mid F_{\ell}\left(\bar{T}, \bar{E}, \overline{C C}^{w}\right)$. These problems are shown to be polynomially solvable (reduction to the assignment problem).

\subsubsection{Minimizing Just-In-Time criteria}

The problem $P \mid d_{i}=d \geq \sum_{i=1}^{n} p_{i}, n$ mit $\mid F_{\ell}(\bar{E}, \bar{T})$ with $F_{\ell}(\bar{E}, \bar{T})=\bar{E}+\bar{T}$ has been tackled by Sundararaghavan and Ahmed [87]. This problem belongs to the class $\mathcal{P}$ and the authors present an optimal algorithm. The problem $P \mid d_{i}=$ $d$, d unknown, nmit $\mid F_{\ell}(\bar{E}, \bar{T})$ with $F_{\ell}(\bar{E}, \bar{T})=\bar{E}+\bar{T}$ is polynomially solvable. Emmons [32] proposes an algorithm to solve it. The problem with machines having 
different non job dependent speeds (proportionnal machines) is also tackled by Emmons, however the complexity of this problem remains open. The problem $P\left|d_{i}=d \geq \sum_{i=1}^{n} p_{i}, n \operatorname{mit}\right| f_{\max }\left(\bar{E}^{w}, \bar{T}^{w}\right)$ with $f_{\max }=\max _{1 \leq i \leq n}\left(w_{i}\left(E_{i}+T_{i}\right)\right)$ is studied by Li and Cheng [58]. The authors show that this problem is strongly $\mathcal{N P}$-hard. Then they propose an $O\left(m n^{2}\right)$ heuristic to solve it. The problem $R \mid d_{i}=d, d$ unknown, $p_{i}=p, n m i t \mid F_{\ell}(\bar{E}, \bar{T}, d)$ is polynomially solvable. Cheng and Chen [25] present an algorithm to solve it.

\subsection{FLOWSHOP SCHEDULING PROBLEMS}

In many manufacturing systems, jobs with multiple operations use machines in the same order. Machines are set up in series and between each machine a queue can be found, in which jobs are waiting for processing. When a job has finished its processing on a machine it goes in the waiting queue of the next machine. Several rules (FIFO for instance) can be used to order the jobs in the queues. The most frequent multicriteria flowshop scheduling problems in literature are about criteria $C_{\max }$ and $\bar{C}$, for two machines or $m$ machines.

\subsubsection{Minimizing completion times of jobs}

The problem $F 2 \mid$ permu $\mid \operatorname{Lex}\left(C_{\max }, \bar{C}\right)$ is strongly $\mathcal{N} \mathcal{P}$-hard. Rajendran [71] presents two heuristics and a branch-and-bound algorithm. This problem has also been tackled by Neppalli et al. [67] who propose a genetic algorithm. Recently, Gupta et al. [45] designed nine heuristic algorithms. All these heuristics outperform Rajendran's ones. Besides, T'kindt et al. [13,93] propose two heuristics that outperform Rajendran's heuristics. They also propose branch-and-bound and dynamic programming algorithms. The problem $F 2 \mid$ permu $\mid F_{\ell}\left(C_{\max }, \bar{C}\right)$ is strongly $\mathcal{N} \mathcal{P}$-hard. Nagar et al. [65] and Serifoglu and Ulusoy [81] propose some heuristics and branch-and-bound algorithms. The problem $F 2 \mid$ permu $\mid \epsilon\left(\bar{C} / C_{\max }\right)$ is strongly $\mathcal{N} \mathcal{P}$-hard and is considered by Sayin and Karabati [76]. They propose a branch-and-bound algorithm that computes the set $E$. For the problem $F \mid$ permu $\mid$ Lex $\left(C_{\max }, \bar{C}\right)$, Selen and Hott [78] and Wilson [104] propose mixed integer models. The problem $F \mid$ permu $\mid C_{\max }, \bar{C}$ has been tackled by Gangadharan and Rajendran [40] and Rajendran [72,73] for which they propose different heuristics. Some bicriteria hybrid flowshop problems are tackled by Riane et al. [74]. These ones are $\mathcal{N} \mathcal{P}$-hard and can be denoted by $F H k,\left(P M^{(l)}\right)_{l=1}^{k} \| F_{\ell}\left(C_{\max }, \bar{C}\right)$ and $F H k,\left(P M^{(l)}\right)_{l=1}^{k} \| \epsilon\left(\bar{C} / C_{\max }\right)$ (see [101]). Two mixed integer programs and some heuristics are presented. The problem $F 2 \mid$ permu, $d_{i} \mid \#\left(C_{\max }, T_{\max }\right)$ is $\mathcal{N} \mathcal{P}$ hard. Daniels and Chambers [26] present a branch-and-bound algorithm that computes the set $E$. A heuristic is also proposed to approximate this set. In the case where the number of machines is $m$, the authors propose a heuristic to solve the problem $F \mid$ permu, $d_{i} \mid \epsilon\left(T_{\max } / C_{\max }\right)$. The problem $F H k,\left(P M^{(l)}(t)\right)_{l=1}^{k}\left|r_{i}^{(1)}, d_{i}^{(k)}\right|$ $\epsilon\left(C_{\max } / T_{\max }\right)$ with $T_{\max }=0$ has been studied by Vignier et al. [100]. This problem is $\mathcal{N} \mathcal{P}$-hard. At each stage and each time $t$, the number of available ressources belongs to $\left[M^{(l)}-1 ; M^{(l)}\right]$ where $M^{(l)}$ is the number of resources at 
stage $l$. The authors propose a branch-and-bound algorithm. For the problem $F 2 \mid$ permu, $d_{i} \mid \#\left(C_{\max }, \bar{U}\right)$, Liao et al. [59] propose a branch-and-bound algorithm to compute the set $E$. The authors also consider the problem $F 2 \mid$ permu, $d_{i} \mid \#\left(C_{\max }, \bar{T}\right)$ for which they propose a branch and bound algorithm that enumerates the set $E$.

\subsubsection{Minimizing Just-In-Time criteria}

In the context of JIT scheduling, Zegordi et al. [105] study the problem $F \mid$ permu, $d_{i}, n m i t \mid F_{\ell}\left(\bar{E}^{w}, \bar{T}^{w}\right)$. They propose a simulated annealing algorithm to solve this strongly $\mathcal{N} \mathcal{P}$-hard problem.

\section{Conclusion}

In this paper we present a general framework, that allows to deal with multicriteria scheduling problems, according to Decision Aid concepts. Multicriteria optimization literature allows us to define some new objective functions for scheduling problems. Then we present a survey on multicriteria scheduling problems and a notation for the new possible objective functions for multicriteria scheduling problems. An overview of multicriteria scheduling literature shows that the different works mainly deal with the minimization of a convex combination of criteria, or with the minimization of a lexicographical order of criteria. The $\epsilon$-constraint approach is also used in few papers. We note that an important part of the works does not take account of the properties and results related to multicriteria optimization literature. This confirms the interest of bringing together multicriteria optimization and multicriteria scheduling literatures.

Acknowledgements. The authors are very grateful to Professor Christian PROUST, who initiated this work a few years ago. Without him, this paper would have never existed.

\section{REFERENCES}

[1] G. Adamopoulos and C. Pappis, Scheduling jobs with different job-dependent earliness and tardiness penalties using the SLK method. Eur. J. Oper. Res. 88 (1996) 336-344.

[2] M. Ahmed and P. Sundararaghavan, Minimizing the weighted sum of late and early completion penalties in a single machine. IEEE Trans. 22 (1990) 288-290.

[3] D. Alcaide, J. Riera and J. Sicilia, An approach to solve bicriterion flow-shop scheduling problems, in Proc. of the 6th International Workshop on Project Management and Scheduling (PMS'98). Istanbul, Turkey (1998) 151-154.

[4] B. Alidaee and A. Ahmadian, Two parallel machine sequencing problems involving controllable job processing times. Eur. J. Oper. Res. 70 (1993) 335-341.

[5] M. Azizoglu, S.K. Kondakci and M. Koksalan, Bicriteria scheduling: Minimizing flowtime and maximum earliness on a single machine, edited by J. Climaco, Multicriteria Analysis. Springer-Verlag (1997) 279-288. 
[6] M. Azizoglu and S. Webster, Scheduling job families about an unrestricted common due date on a single machine. Internat. J. Production Res. 35 (1997) 1321-1330.

[7] U. Bagchi, Y.-L. Chang and R. Sullivan, Minimizing absolute and squared deviations of completion times with different earliness and tardiness penalties and a common due date. Naval Res. Logist. 34 (1987) 739-751.

[8] U. Bagchi, R. Sullivan and Y.-L. Chang, Minimizing mean absolute deviation of completion times about a common due date. Naval Res. Logist. Quarterly 33 (1986) 227-240.

[9] U. Bagchi, R. Sullivan and Y.-L. Chang, Minimizing mean squared deviation of completion times about a common due date. Management Sci. 33 (1987) 894-906.

[10] S. Bansal, Single machine scheduling to minimize weighted sum of completion times with secondary criterion - a branch and bound approach. Eur. J. Oper. Res. 5 (1980) 177-181.

[11] J. Barnes and L. Vanston, Scheduling jobs with linear delay penalties and sequence dependent setup costs. Oper. Res. 29 (1981) 146-160.

[12] C. Bector, Y. Gupta and M. Gupta, Determination of an optimal common due date and optimal sequence in a single machine job shop. Internat. J. Production Res. 26 (1988) 613-628.

[13] J.-C. Billaut, V. T'kindt, P. Richard and C. Proust, Three exact methods and an efficient heuristic for solving a bicriteria flowshop scheduling problem, in Multiconference on Computational Engineering in Systems Applications (CESA 98), Symposium on Industrial and Manufacturing Systems, IEEE-SMC/IMACS. Nabeul-Hammamet, Tunisia (1998) 371-377.

[14] J. Blazewicz, K. Ecker, E. Pesch, G. Schmidt and J. Weglarz, Scheduling computer and manufacturing processes. Springer-Verlag (1996).

[15] V. Bourgade, L. Aguilera, B. Penz and Z. Binder, Problème industriel d'ordonnancement bicritère sur machine unique : modélisation et aide à la décision. APII 29 (1995) 331-341.

[16] V. Bowman, On the relationship of the tchebycheff norm and the efficient frontier of multiple-criteria objectives, edited by H. Thiriez and S. Zionts, Multiple Criteria Decision Making. Springer-Verlag (1976) 76-85.

[17] R. Burns, Scheduling to minimize the weighted sum of completion times with secondary criteria. Naval Res. Logist. Quarterly 23 (1976) 125-129.

[18] S. Chand and H. Schneeberger, Single machine scheduling to minimize weighted completion time with maximum allowable tardiness, Research report. University of Purdue (1984).

[19] S. Chand and H. Schneeberger, A note on the single machine scheduling problem with minimum weighted completion time and maximum allowable tardiness. Naval Res. Logist. Quarterly 33 (1986) 551-557.

[20] S. Chand and H. Schneeberger, Single machine scheduling to minimize weighted earliness subject to no tardy jobs. Eur. J. Oper. Res. 34 (1988) 221-230.

[21] C.-L. Chen and R. Bulfin, Scheduling unit processing time jobs on a single machine with multiple criteria. Comput. Oper. Res. 17 (1990) 1-7.

[22] C.-L. Chen and R. Bulfin, Complexity of single machine, multi-criteria scheduling problems. Eur. J. Oper. Res. 70 (1993) 115-125.

[23] C.-L. Chen and R. Bulfin, Complexity of multiple machines, multi-criteria scheduling problems, in 3rd Industrial Engineering Research Conference (IERC'94). Atlanta, USA (1994) $662-665$.

[24] Z.-L. Chen, Scheduling and common due date assignment with earliness and tardiness penalties and batch delivery costs. Eur. J. Oper. Res. 93 (1996) 49-60.

[25] T. Cheng and Z.-L. Chen, Parallel-machine scheduling problems with earliness and tardiness penalties. J. Oper. Res. Soc. 45 (1994) 685-695.

[26] R. Daniels and R. Chambers, Multiobjective flow-shop scheduling. Naval Res. Logist. 37 (1990) 981-995.

[27] P. Dileepan and T. Sen, Bicriterion static scheduling research for a single machine. Omega 16 (1998) 53-59.

[28] P. Dileepan and T. Sen, Bicriterion jobshop scheduling with total flowtime and sum of squared lateness. Engrg. Costs and Production Economics 21 (1991) 295-299. 
[29] M. Ehrgott, Multiple Criteria Optimization: Classification and Methodology, Ph.D. Thesis. University of Kaiserslautern, Germany, in English (1997).

[30] H. Emmons, A note on a scheduling problem with dual criteria. Naval Res. Logist. Quarterly 22 (1975) 615-616.

[31] H. Emmons, One machine sequencing to minimize mean flow time with minimum number tardy. Naval Res. Logist. Quarterly 22 (1975) 585-592.

[32] H. Emmons, Scheduling to a common due date on parallel uniform processors. Naval Res. Logist. 34 (1987) 803-810.

[33] G. Evans, An overview of techniques for solving multiobjective mathematical programs. Management Sci. 30 (1984) 1268-1282.

[34] T. Fry, R. Armstrong and R. Blackstone, Minimizing weighted absolute deviation in single machine scheduling. IEEE Trans. 19 (1987) 445-450.

[35] T. Fry, R. Armstrong and H. Lewis, A framework for single machine multiple objective sequencing research. Omega 17 (1989) 595-607.

[36] T. Fry and R. Blackstone, Planning for idle time: A rationale for underutilization of capacity. Int. J. Prod. Res. 26 (1988) 1853-1859.

[37] T. Fry and G. Leong, Bi-criterion single-machine scheduling with forbidden early shipments. Engrg. Costs and Production Sci. 10 (1986) 133-137.

[38] T. Fry and G. Leong, A bi-criterion approach to minimizing inventory costs on a single machine when early shipments are forbidden. Comput. Operat. Res. 14 (1987) 363-368.

[39] T. Fry, G. Leong and T. Rakes, Single machine scheduling: A comparison of two solution procedures. Omega 15 (1987) 277-282.

[40] R. Gangadharan and C. Rajendran, A simulated annealing heuristic for scheduling in a flowshop with bicriteria. Comput. Industrial Engrg. 27 (1994) 473-476.

[41] M. Garey, R. Tarjan and G. Wilfong, One-processor scheduling with symmetric earliness and tardiness penalties. Math. Oper. Res. 13 (1988) 330-348.

[42] F. Gembicki, Vector Optimization for Control with Performance and Parameter Sensitivity Indices, Ph.D. Thesis. Case Western Reserve University, Cleveland, USA (1973).

[43] A. Geoffrion, Proper efficiency and the theory of vector maximization. J. Math. Anal. Appl. 22 (1968) 618-630.

[44] J. Gupta, J. Ho and A. VanderVeen, Single machine hierarchical scheduling with customer orders and multiple job classes. Ann. Oper. Res. 70 (1997) 127-143.

[45] J. Gupta, V. Neppalli and F. Werner, Minimizing total flow time in a two-machine flowshop problem with minimum makespan. Internat. J. Production Economics (to appear).

[46] S. Gupta and T. Sen, Minimizing a quadratic function of job lateness on a single machine. Engrg. Costs and Production Economic 7 (1983) 187-194.

[47] Y. Haimes, L. Ladson and D. Wismer, On a bicriterion formulation of the problems of integrated system identification and system optimization. IEEE Trans. Systems, Man and Cybernetics 1 (1971) 296-297.

[48] H. Heck and S. Roberts, A note on the extension of a result on scheduling with secondary criteria. Naval Res. Logist. Quarterly 19 (1972) 4.

[49] J. Hoogeveen, Single-Machine Bicriteria Scheduling, Ph.D. Thesis. CWI Amsterdam (1992).

[50] J. Hoogeveen and S. VandeVelde, Minimizing total completion time and maximum cost simultaneously is solvable in polynomial time. Oper. Res. Lett. 17 (1995) 205-208.

[51] T. John, Tradeoff solutions in single machine production scheduling for minimizing flow time and maximum penalty. Comput. Oper. Res. 16 (1984) 471-479.

[52] J. Kanet, Minimizing the average deviation of job completion times about a common due date. Naval Res. Logist. Quarterly 28 (1981) 643-651.

[53] S. Kondakci, E. Emre and M. Koksalan, Scheduling of unit processing time jobs on a single machine, edited by G. Fandel and T. Gal, Multiple Criteria Decision Making. SpringerVerlag, Lecture Notes in Econom. and Math. Systems (1997) 654-660.

[54] C. Koulamas, Single-machine scheduling with time windows and earliness/tardiness penalties. Eur. J. Oper. Res. 91 (1996) 190-202. 
[55] S. Lakshminarayan, R. Lakshmanan, R. Papineau and R. Rochette, Optimal single-machine scheduling with earliness and tardiness penalties. Oper. Res. 26 (1978) 1079-1082.

[56] C.-Y. Lee and G. Vairaktarakis, Complexity of single machine hierarchical scheduling: $A$ survey, edited by P.M. Pardalos, Complexity in Numerical Optimization. World Scientific Publishing Co. (1993) 269-298.

[57] J.-T. Leung and G. Young, Minimizing schedule length subject to minimum flow time. SIAM J. Comput. 18 (1989) 314-326.

[58] C.-L. Li and T. Cheng, The parallel machine min-max weighted absolute lateness scheduling problem. Naval Res. Logist. 41 (1994) 33-46.

[59] C.-J. Liao, W.-C. Yu and C.-B. Joe, Bicriterion scheduling in the two-machine flowshop. J. Oper. Res. Soc. 48 (1997) 929-935.

[60] K. Lin, Hybrid algorithm for sequencing with bicriteria. J. Opt. Theor. Appl. 39 (1983) 105-124.

[61] S. McCormick and M. Pinedo, Scheduling $\mathrm{n}$ independant jobs on $\mathrm{m}$ uniform machines with both flowtime and makespan objectives: A parametric analysis. ORSA J. Comput. 7 (1995) 63-77.

[62] K. Miettinen. On the Methodology of Multiobjective Optimization with Applications, Ph.D. Thesis. University of Jyvaskyla, Department of Mathematics (1994).

[63] S. Miyazaki, One machine scheduling problem with dual criteria. J. Oper. Res. Soc. Jpn. 24 (1981) 37-50.

[64] A. Nagar, J. Haddock and S. Heragu, Multiple and bicriteria scheduling: A literature survey. Eur. J. Oper. Res. (1995) 88-104.

[65] A. Nagar, S. Heragu and J. Haddock, A branch-and-bound approach for a two-machine flowshop scheduling problem. J. Oper. Res. Soc. 46 (1995) 721-734.

[66] R. Nelson, R. Sarin and R. Daniels, Scheduling with multiple performance measures: The one-machine case. Management Sci. 32 (1986) 464-479.

[67] V. Neppalli, C.-L. Chen and J. Gupta, Genetic algorithms for the two-stage bicriteria flowshop problem. Eur. J. Oper. Res. 95 (1996) 356-373.

[68] P. Ow and T. Morton, Filtered beam search in scheduling. Internat. J. Production Res. 26 (1988) 35-62.

[69] P. Ow and T. Morton, The single machine early/tardy problem. Management Sci. 35 (1989) 177-190.

[70] S. Panwalker, M. Smith and A. Seidmann, Common due date assignment to minimize total penalty for the one machine scheduling problem. Oper. Res. 30 (1982) 391-399.

[71] C. Rajendran, Two-stage flowshop scheduling problem with bicriteria. J. Oper. Res. Soc. 43 (1992) 871-884.

[72] C. Rajendran, A heuristic for scheduling in flowshop and flowline-based manufacturing cell with multi-criteria. Internat. J. Production Res. 32 (1994) 2541-2558.

[73] C. Rajendran, Heuristics for scheduling in flowshop with multiple objectives. Eur. J. Oper. Res. 82 (1995) 540-555.

[74] F. Riane, N. Meskens and A. Artiba, Bicriteria scheduling hybrid flowshop problems, in International Conference on Industrial Engineering and Production Managment (IEPM'97). Lyon, France (1997) 34-43.

[75] B. Roy, Méthodologie multicritère d'aide à la décision. Economica (1985).

[76] S. Sayin and S. Karabati, A bicriteria approach to the two-machine flow shop scheduling problem. Eur. J. Oper. Res. 113 (1999) 435-449

[77] A. Seidmann, S. Panwalker and M. Smith, Optimal assignment of due-dates for a single processor scheduling problem. Internat. J. Production Res. 19 (1981) 393-399.

[78] W. Selen and D. Hott, A mixed integer goal-programming formulation of a flowshop scheduling problem. J. Oper. Res. Soc. 37 (1986) 1121-1128.

[79] T. Sen and S. Gupta, A branch-and-bound procedure to solve a bicriterion scheduling problem. IEEE Trans. 15 (1983) 84-88. 
[80] T. Sen, F. Raiszadeh and P. Dileepan, A branch-and-bound approach to the bicriterion scheduling problem involving total flowtime and range of lateness. Management Sci. 34 (1988) 255-260.

[81] F. Serifoglu and G. Ulusoy, A bicriteria two-machine permutation flowshop problem. Eur. J. Oper. Res. 107 (1998) 414-430.

[82] J. Shantikumar, Scheduling $n$ jobs on one machine to minimize the maxium tardiness with minimum number tardy. Comput. Oper. Res. 10 (1983) 255-266.

[83] J. Sidney, Optimal single-machine scheduling with earliness and tardiness penalties. Oper. Res. 25 (1977) 62-69.

[84] W. Smith, Various optimizers for single-stage production. Naval Res. Logist. Quarterly 3 (1956) 59-66.

[85] R. Soland, Multicriteria optimization: A general characterization of efficient solutions. Decision Sci. 10 (1979) 27-38.

[86] R. Steuer, Multiple criteria optimization: Theory, computation and application. Wiley (1986).

[87] P. Sundararaghavan and M. Ahmed, Minimizing the sum of absolute lateness in singlemachine and multimachine scheduling. Naval Res. Logist. Quarterly 31 (1984) 325-333.

[88] W. Szwarc, Single-machine scheduling to minimize absolute deviation of completion times from a common due date. Naval Res. Logist. 36 (1989) 663-673.

[89] V. T'kindt and J.-C. Billaut, L'ordonnancement multicritère. Presses de l'Université de Tours (2000).

[90] V. T'kindt, J.-C. Billaut and H. Houngbossa, A multi-criteria heuristic to solve a 2-stage hybrid flowshop scheduling problem. Eur. J. Automation (JESA) 34 (2000) 1187-1200.

[91] V. T'kindt, J.-C. Billaut, S. Laurin and O. Meslet, Un algorithme optimal polynomial pour résoudre un problème d'ordonnancement bicritère à machines parallèles, in Conference on Automation-Computers Engineering-Image-Signal (AGIS'97). Angers, France (1997) 179184.

[92] V. T'kindt, J.-C. Billaut and C. Proust, Solving a bicriteria scheduling problem on unrelated parallel machines occuring in the glass bottle industry. Eur. J. Oper. Res. 135 (2001) $42-49$.

[93] V. T'kindt, P. Richard, C. Proust and J.-C. Billaut, Resolution of a 2-machine bicriteria flowshop scheduling problem, in Int. Conference on Methods and Applications of Multicriteria Decision Making (MAMDM'97). Mons, Belgium (1997) 139-143.

[94] M. VandenAkker, H. Hoogeveen and S. VandeVelde, in 6th International Workshop on Project Management and Scheduling (PMS'98). Istanbul, Turkey (1998).

[95] L. VanWassenhove and K. Baker, A bicriterion approach to time/cost trade-offs in sequencing. Eur. J. Oper. Res. 11 (1982) 48-54.

[96] L. VanWassenhove and L. Gelders, Four solution techniques for a general one machine scheduling problem: A comparative study. Eur. J. Oper. Res. 2 (1978) 281-290.

[97] L. VanWassenhove and L. Gelders, Solving a bicriterion scheduling problem. Eur. J. Oper. Res. 4 (1980) 42-48.

[98] R. Vickson, Choosing the job sequence and processing times to minimize total processing plus flow cost on a single machine. Oper. Res. 28 (1980) 115-167.

[99] R. Vickson, Two single machine sequencing problems involving controllable job processing times. IEEE Trans. 12 (1980) 158-162.

[100] A. Vignier, J.-C. Billaut and C. Proust, Solving $k$-stage hybrid flowshop scheduling problems, in Multiconference on Computational Engineering in Systems Applications (CESA'96), Symposium on Discrete Events and Manufacturing Systems (IEEESMC/IMACS). Lille, France (1996) 250-258.

[101] A. Vignier, J.-C. Billaut and C. Proust, Les flowshop hybrides : état de l'art. RAIRO: Oper. Res. 33 (1999) 117-183.

[102] S. Webster, P. Job and A. Gupta, A genetic algorithm for scheduling job families on a single machine with arbitrary earliness/tardiness penalties and an unrestricted common due date. Internat. J. Production Res. 36 (1998) 2543-2551. 
[103] A. Wierzbicki, The use of reference objectives in multiobjective optimization, edited by G. Fandel and T. Gal, Multiple criteria decision making, theory and application. SpringerVerlag (1990) 468-486.

[104] J. Wilson, Alternative formulations of a flow-shop scheduling problem. J. Oper. Res. Soc. 40 (1989) 395-399.

[105] S. Zegordi, K. Itoh and T. Enkawa, A knowledgeable simulated annealing scheme for the early/tardy flow shop scheduling problem. Internat. J. Production Res. 33 (1995) 14491466 .

to access this journal online: www.edpsciences.org 